\title{
Automatic Ocular Alignment Evaluation for Strabismus Detection Using Index of Phylogenetic Diversity and Support Vector Machine
}

\author{
Thayane de O. Simões, Aristófanes C. Silva, Johnatan C. Souza, João D. S. Almeida, Anselmo C. Paiva \\ Núcleo de Computação Aplicada (NCA) \\ Universidade Federal do Maranhão (UFMA), São Luís - Brasil \\ Email: \{thayanesimoes19, johnatancarvalho06\}@gmail.com,
}

\{ari, jdallyson, paiva\}@nca.ufma.br

\begin{abstract}
Strabismus is a pathology that affects the parallelism between two eyes. Estimates show that $1.3 \%$ to $3.5 \%$ of children aged six months to six years have strabismus around the world. Strabismus can lead to irreversible loss of vision, so early detection and appropriate treatment increase the likelihood of alignment being restored to normal. That said, in this study we proposed an automatic evaluation system to detect strabismus in face images, analyzing the horizontal positioning of the limb center in relation to the center of the corners of the eyes. The proposed method consists of five steps: (1) acquisition, (2) detection of the eye region, (3) segmentation and reconstruction of the sclera, (4) detection of the limb region and corners of the eyes and (5) identification of the presence of strabismus, with this approach we obtain $90.1 \%$ sensitivity, $100 \%$ specificity and 91.1\% accuracy.
\end{abstract}

Keywords-Strabismus Detection, Hirschberg test, Index of Phylogenetic Diversity, SegNet

\section{INTRODUÇÃO}

O estrabismo é uma anomalia caracterizada por um desequilíbrio nos músculos oculares, fazendo com que os dois olhos não fixem o objeto ao mesmo tempo. Enquanto um dos olhos se fixa num ponto frontal, o outro pode se desviar horizontalmente para dentro (estrabismo convergente) ou para fora (estrabismo divergente) e para cima ou para baixo (estrabismo vertical). Dessa forma, o cérebro recebe duas imagens com focos diferentes, em vez de duas imagens que devem se fundir numa única imagem [1]. Essa anomalia pode ser classificada de acordo com a direção do foco em que o olho desviado aponta, identificado olhando para o paciente ou latente, quando requer testes para obter o diagnóstico [2].

Para diagnosticar o estrabismo, vários testes são realizados atualmente. Por exemplo, o teste de acuidade visual que avalia as mudanças na visão a curta e longa distância; o exame de fundo de olho que observa a estrutura física do olho para verificar a presença de outras doenças; o cover teste que mede o desvio e o movimento dos olhos e o teste de Hirschberg que é usado para determinar a extensão do erro de refração, que é útil na decisão da potência da lente necessária para compensar as mudanças [3], esse teste consiste em incidir um pequeno foco de luz nos olhos do paciente e medir o ângulo kappa, que é gerado devido ao desalinhamento entre os eixos visual e pupilar, no entanto seu diagnostico pode ser impreciso pois assume uma regra geral e depende muito da avaliação subjetiva do médico [4]. Diante disso, o estrabismo deve ser diagnosticado precocemente para que seu tratamento seja eficiente e aumente a probabilidade do alinhamento ser restaurado ao normal e evite consequências como baixa visão, podendo progredir para a cegueira.

Este trabalho propõe uma nova abordagem computacional para detectar a presença de estrabismo, utilizando fotografia da face, na qual analisa-se automaticamente a diferença entre o centro do limbo em relação aos cantos dos olhos, utilizando índices de diversidade filogenética para segmentação da esclera e deep learning, a fim de oferecer uma segunda opinião aos especialistas, servindo como apoio para tomada de decisões.

\section{TRABALhOS RELACIONADOS}

$\mathrm{Na}$ literatura, existem algumas pesquisas no contexto desse trabalho. Abaixo, enumera-se alguns deles

Em Almeida et al. (2015) [5] propôs uma metodologia para detectar e diagnosticar estrabismo usando o teste de Hirschberg: (1) segmentação facial, (2) deteç̧ão da região ocular, (3) localização de olho, (4) localização ao do brilho e (5) diagnóstico de estrabismo. Os pesquisadores utilizaram 200 imagens de 40 pacientes, obtidas em cinco posições diferentes do olhar. Com relação a detecção de estrabismo, este estudo obteve uma acurácia de $88 \%$ na posição $\mathrm{PP}, 93,34 \%$ na posição INFRA, $77,41 \%$ na posição SUPRA, $80 \%$ na posição LEVO e $81,48 \%$ na posição DEXTRO. Quanto ao diagnóstico, o melhor resultado foi no diagnóstico de esotropia (ET) obtendo $100 \%$ de acurácia.

Em Valente et al. (2017) [2] propuseram uma metodologia para diagnosticar automaticamente o estrabismo em vídeos através do cover test em pacientes com exotropia, e é dividido em quatro etapas: (1) detecção dos olhos, (2) localização do limbo, (3) rastreamento do movimento dos olhos e (4) detecção do oclusor. Os experimentos foram realizados em 15 vídeos de 7 pacientes, obtendo especificidade de $100 \%$, sensibilidade de $80 \%$ e acurácia de $93,33 \%$. 
Zhen et al. (2019) [3] apresentam uma metodologia para a detecção automática do estrabismo em vídeo através do exame cover test. É proposto um método que analisa o movimento dos olhos que consiste em: (1) detecção da região dos olhos, (2) template matching para localizar a íris, (3) localização da pupíla, (4) cálculo do desvio horizontal e vertical e por fim (5) detecção do estrabismo. O método proposto alcançou $91 \%$ de acurácia na direção horizontal, com erro de 8 dioptrias e $86 \%$ de acurácia na direção vertical, com um erro de 4 dioptrias.

Estes são alguns dos métodos de detecção de estrabismo mais presentes na literatura. Podemos observar três pontos importantes em comum entre esses trabalhos, quais sejam: necessidade de uma etapa de localização dos olhos; localização do limbo ${ }^{1}$; e as técnicas usadas em cada um desses trabalhos são fortemente dependentes do conjunto de dados da imagem. Em nosso método, tentamos superar essa limitação identificando a presença do estrabismo para qualquer base de imagens.

\section{Metodologia Proposta}

A metodologia proposta consiste nas seguintes etapas: aquisição da base de imagens, pré-processamento, segmentação e reconstrução da esclera, localização do limbo e cantos dos olhos e, por fim, a classificação em estrábico ou normal. A Figura 1 ilustra as etapas da metodologia.

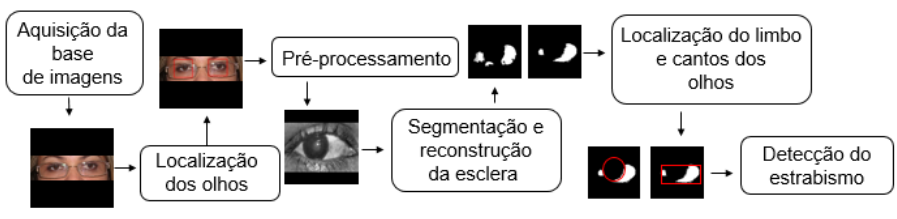

Fig. 1. Metodologia proposta.

\section{A. Bases de imagens}

Este trabalho utiliza as bases de imagens UBIRIS.v2 [6], a base privada SSRBC 2017 [7] e a base privada de estrabismo utilizada por Almeida et al. [5].

As bases de imagens utilizadas para realizar o treinamento na etapa de segmentação da esclera foram a UBIRIS.v2 e SSRBC 2017. A base UBIRIS.v2 contém 201 imagens com 400 pixels de largura e 300 pixels de altura, enquanto que o conjunto de imagens do SSRBC 2017 possui 120 imagens oculares de 30 indivíduos.

Já nos testes, foram utilizadas as imagens da base privada de estrabismo que foram adquiridas num consultório oftalmológico em São Luís-MA, contendo 45 pacientes, sendo que cada paciente contém 5 posições diferentes: posição primária do olhar (PPO), posição do olhar direcionado para esquerda (LEVO), posição do olhar direcionado para direita (DEXTRO), posição do olhar direcionado para baixo (INFRA) e posição do olhar direcionado para cima (SUPRA) [5]. A Figura 2 apresenta um exemplo da base.

\footnotetext{
${ }^{1}$ estrutura de transição entre a córnea e a esclera
}

A base contém 225 imagens divididas em duas classes: 40 pacientes com estrabismos e 5 pacientes normais e, para cada paciente, há marcações da esclera, marcações do limbo e o diagnóstico do especialista. (b)

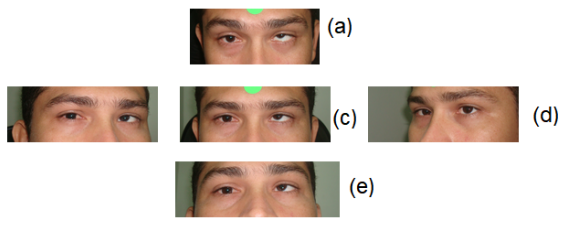

Fig. 2. Imagens nas 5 posições do olhar: (a) SUPRA, (b) DEXTRO, (c) PPO, (d) LEVO, (e) INFRA [5].

\section{B. Localização dos olhos}

Nesta etapa, para detectar a região dos olhos, foi utilizada a marcação da esclera disponibilizada na base Almeida et al. [5], pois o estudo não objetivou realizar a detecção dos olhos, visto que já existem diversos trabalhos que tratam desse problema, como os trabalhos de Lu et al. (2018) [8] e Lucio et al. (2018) [9] que utilizam redes neurais convolucionais para tal fim. A partir da Figura 3(a) foi possível localizar os olhos e mapear essa região na imagem original conforme a Figura 3 (b).

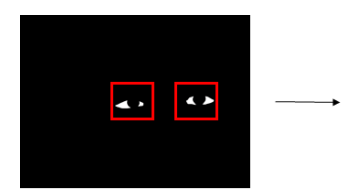

(a)

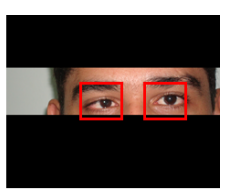

(b)
Fig. 3. (a) Marcação da esclera, (b) Localização dos olhos.

\section{Segmentação e Reconstrução da esclera}

A esclera é região branca dos olhos, ao redor do globo ocular e contém vasos sanguíneos distribuídos aleatoriamente. Devido ao posicionamento humano, múltiplas direções do olhar e variações de iluminação resultam em desafios para realizar uma segmentação considerada adequada.

Após a localização dos olhos, a etapa de segmentação da esclera foi dividida em três subetapas: (1) pré-processamento, (2) divisão da imagem em patches, extração de características e (3) classificação dos patches em esclera e não esclera usando Support Vector Machine (SVM).

1) Pré-processamento: Nesta etapa, a imagem colorida é transformada numa imagem de nível de cinza, onde a esclera é destacada. Para diferenciar a esclera das outras regiões da imagem, realiza-se a fusão dos três canais com uma baixa contribuição do canal vermelho, pois em comparação com os demais canais, o canal vermelho possui maior intensidade [10].

A imagem de entrada é composta por cada canal $R, G$ e B separadamente, onde cada canal (C) é mapeado em um intervalo entre 0 e 1 por meio da transformação linear baseada em uma função quase sigmoide definida em [11], conforme a Equação 1:

$$
C^{\prime}=1-b^{\frac{c}{\mu}} / a b^{\frac{c}{\mu}}+1
$$


onde $a$ e $b$ sendo pré-definidos com valores constantes de $a=2+\sqrt{3}$ e $b=7-4 \sqrt{3}$. A escolha do $\mu$ assume uma regra fundamental para evitar a possibilidade de valores de intensidade maiores e são mapeados para o intervalo de [0,1], como mostra a Equação 2:

$$
\begin{array}{r}
\mu=\min (n R, n G, n B) \\
n R=\operatorname{mean}(R)+\sigma(R) / 2 \\
n G=\operatorname{mean}(G)+\sigma(G) / 2 \\
n B=\operatorname{mean}(B)+\sigma(B) / 2
\end{array}
$$

onde $\sigma(R), \sigma(G)$ e $\sigma(B)$ representam o desvio padrão dos valores de intensidade nos canais RGB. Em seguida, obtémse uma imagem em nível de cinza combinando os canais normalizados de acordo com Equação 3 e finalmente essa nova imagem é normalizada no histograma stretching, assumindo valores de 0 a 255 .

$$
\begin{array}{r}
I q=G^{\prime}+B^{\prime}-R^{\prime} \\
I=255 *(I q-\min (I q) / \max (I q)-\min (I q))
\end{array}
$$

2) Extração de características: Primeiramente, nesta etapa, as imagens de treino e teste foram divididas patches. As imagens obtidas da base SSRBC 2017 [7] foram utilizadas para a construção da base de treino. Nessa base, rotulamos duas classes: esclera e não-esclera, o critério utilizado para essa classificação e baseado na proporção de pixels que pertecem ou não à esclera, como mostra a Figura 4.

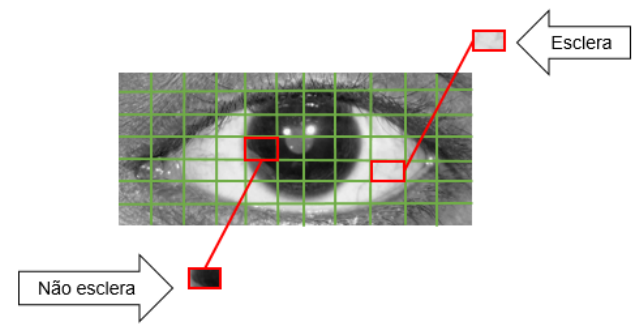

Fig. 4. Divisão da imagem em patches na base SSRBC 2017 [7].

Em seguida, realizou-se a extração de características usando índices de diversidade filogenética, baseados na análise de textura e, posteriormente, a classificação dos pixels em esclera e não-esclera com base nessas características.

A filogenia é representada por árvores filogenéticas, onde essas árvores devem expressar similaridade, ancestralidade ou parentescos evolutivos entre espécies. Nelas, as folhas representam os organismos e os nós internos correspondem aos seus ancestrais hipotéticos, como apresenta a Figura 5. As árvores filogenéticas, combinadas com os índices de diversidade filogenética, são usadas na biologia para comparar os padrões de comportamento das espécies em diferentes áreas.

A Figura 5 apresenta uma comparação entre os termos de biologia e da metodologia proposta, sendo que, em imagens, as espécies representam os níveis de cinza, os indivíduos representam a quantidade de pixels.

Para tanto, na literatura os índices de diversidade filogenética são utilizados para extrair características em nódulos

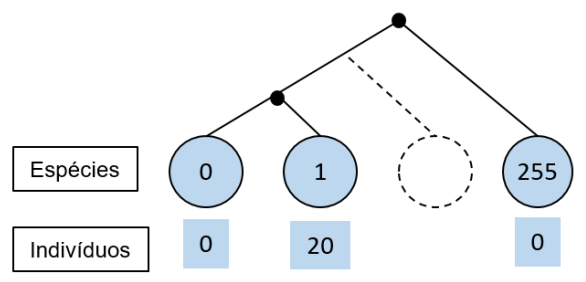

Fig. 5. Árvore enraizada em forma de cladograma inclinada.

pulmonares [12] [13] e na diferenciação de nódulos benignos e malignos [14] [15] [16]. Diferentemente da lteratura, nossa metodolgia extrai informações dos patches para classificar em esclera ou não-esclera, gerando assim a segmentação da região de interesse. Assim, os índices são divididos em quatro grupos: (1) baseados na distância entre pares de espécies; (2) baseados em riqueza de espécies; (3) baseados na topologia; e (4) baseados em caminho mínimo.

a) Índices de Diversidade Filogenética Baseados na Distância entre Pares de Espécies: Esses índices são baseados na matriz de distância entre todas as espécies da comunidade, com isso, as distâncias são representadas pelo comprimento dos ramos das árvores filogenéticas. Os índices pertencentes a esse grupo são: entropia quadrática intensiva [17], entropia quadrática extensiva [17], distinção taxonômica total [18] e distinção taxonômica média [19].

A entropia quadrática intensiva (I) [17] estabelece uma ligação entre os índices de biodiversidade e índices de diversidade e a entropia quadrática extensiva (J) [17] representa a soma das diferenças das espécies, como apresentam as Equações 4 e 5 respectivamente.

$$
I=\left[\sum d_{i, j}\right] / s^{2} \quad \text { (4) } \quad J=\sum d_{i, j}
$$

onde $d_{i, j}$ representa a distância entre as espécies $i$ e $j$, e $s$ representa o número de espécies.

A distinção taxonômica média (DTM) refere-se à distância taxonômica média entre duas espécies [18] e a distinção taxonômica total (DTT) representa a distinção taxonômica média de todas as espécies dentro da comunidade. Esse índices são respectivamente, definidos nas Equações 6 e 7.

$$
\begin{aligned}
D T M & =\left[\sum \sum_{i<j} d_{i, j}\right] /[s(s-1) / 2] \\
D T T & =\sum_{i}\left[\left(\sum_{i \neq j} d_{i, j}\right)(s-1)\right]
\end{aligned}
$$

onde $d_{i, j}$ representa a distância entre as espécies $i$ e $j$ e o número de espécies é definido por $s$.

b) Índices de Diversidade Filogenética Baseados em Riqueza de Espécies: : Esses índices permitem medir a relação entre dois indivíduos escolhidos aleatoriamente na comunidade, por meio dos índices de diversidade taxonômica e distinção taxonômica [20].

$\mathrm{O}$ índice de diversidade taxonômica $(\Delta)$ representa a abundância das espécies e a relação taxonômica entre eles e 
o índice de distinção taxonômica $(\Delta *)$ representa a distância taxonômica média entre dois indivíduos de espécies diferentes. Estes índices são definidos pelas Equações 8 e 9.

$$
\begin{aligned}
\Delta & =\sum \sum_{i<j} w_{i, j} x_{i} x_{j} /[n(n-1) / 2] \\
\Delta^{*} & =\sum \sum_{i<j} w_{i, j} x_{i} x_{j} / \sum \sum_{i<j} x_{i} x_{j}
\end{aligned}
$$

onde $w_{i}$ é a distância entre as espécies $i$ e $j, x_{i}$ e $x_{j}$ são respectivamente a quantidade de indivíduos da $i$-ésima e $j$ ésima espécie e $n$ é o número total de indivíduos.

c) Índices de Diversidade Filogenética Baseados na Topologia: Segundo Vane-Wright et al.. [21] esses índices refletem a ordem de ramificação filogenética dentro de um grupo, sendo que cada espécie de uma comunidade é ponderada de acordo com o número de nós entre as espécies e a raiz da árvore filogenética, ou seja, as espécies com pesos maiores são aquelas com maiores distâncias na raiz. Os índices que compõem esse grupo são: a soma básica dos pesos (Q) e a soma dos pesos normalizados (W) [22].

$\mathrm{O}$ índice $\mathrm{Q}$ e o quociente de todos os nós dividido pelo número de nós entre a raiz de uma espécie representa a soma das contribuiçõos de cada espécie para a diversidade, e o índice W representa para cada espécie o peso normalizado, ou seja, os valores de $\mathrm{Q}$ de cada espécie dividido pelo valor de $Q_{\text {min }}$. Esses índices são representados pelas Equações 10 e 11 , respectivamente.

$$
\begin{gathered}
Q=\sum Q_{i} \\
Q_{i}=I / I_{i} \\
I=\sum I_{i}
\end{gathered}
$$

$$
\begin{array}{r}
W=\sum W_{i} \\
W_{i}=Q_{i} / Q_{\text {min }}
\end{array}
$$

onde $Q_{i}$ é o quociente do total de nós da raiz para todas as espécies por $I_{i}$, que é o númerode nós entre a raiz e a espécie $i$, e $Q_{\min }$ representa o quociente do caminho mínimo da raiz para a espécie.

d) Indices de Diversidade Filogenética Baseados em Caminho Mínimo: Esses índices analisam a distância entre as espécies e a comunidade. Então, quanto menor o caminho, menor será a quantidade de espécies e logo será menor a diversidade. Os índices pertencentes a esse grupo são: diversidade filogenética $\left(P D_{N O D E}\right)$ [23] e a soma das distâncias filogenéticas $\left(P D_{R O O T}\right)$ [24].

O índice $P D_{N O D E}$ na biologia é obtido pela soma dos comprimentos dos braços da árvore filogenética das espécies de uma comunidade multiplicado pelo número de pares das espécies [23], conforme apresenta a Equação 12.

$$
\begin{array}{r}
A_{i}=\left(\sum I_{i} / D_{\text {ind }_{i j}}\right) \\
C_{1}=\sum D_{m} * A_{i} \\
C_{2}=\sum A_{i} \\
P D_{N O D E}=C_{1} / C_{2}
\end{array}
$$

onde $D_{m}$ representa o somatório da distância no momento da interação e $D_{i n d_{i j}}$ representa a distância individual em cada interação.

O índice $P D_{R O O T}$ é a soma das distâncias filogenéticas entre cada par de espécies multiplicado pelo número de pares das espécies, como apresenta a Equação 13.

$$
P D_{R O O T}=(n(n-1) / 2) * \frac{\Delta}{\Delta^{*}}
$$

onde $n$ é o número total de indivíduos.

3) Reconstrução da esclera: Observamos que, após a classificação dos paches utilizando os índices de diversidade filogenética para a segmentação ao da esclera, parte das escleras estavam sendo perdidas e,por essa razão, outra etapa era necessária para melhorar este resultado inicial. Portanto, foi' modificada a rede ResNet-18 [25] baseado na rede segmentada em Ehsani et al. (2018) [26], que anteriormente era usado para reconstruir objetos oclusos em imagens.

Para o treinamento da rede de reconstrução, utilizamos a saída do último resblock da ResNet-18 e a passamos para uma camada totalmente conectada para realizar o upsampling. Em seguida, a saída da camada totalmente conectada e passada para uma camada de agrupamento final máxima e em nossa rede de reconstrução usamos batch normalization [27] logo após cada camada convolucional. Para as camadas máximas de pooling foram usados kernels de tamanho $3 \times 3$ com stride 2 , resultando assim na reconstrução das regiões da esclera.

\section{Localização do limbo e cantos dos olhos}

A localização do limbo e realizado através da Transformada de Hough (HT) para detectar círculos, sendo que este é um dos métodos matemáticos mais utilizados para detecção de elementos que podem ser parametrizados (linhas, círculos, elipses) [28]. A eficiência do HT depende da localização ideal das bordas do objeto, portanto, o HT é aplicado à imagem original com os parâmetros proporcionais ao raio do limbo, com a região do limbo encontrada na mesma e na esclera segmentada.

Para localizar os cantos dos olhos foi criado uma Bounding Box nBox na esclera segmentada, esta é uma técnica que desenha o menor retângulo que encaixe num determinado objeto. No entanto, há casos em que não foi possível detectar os cantos dos olhos utilizando a esclera como referência, então nesses casos o limbo é utilizado como referência. Assim, a localização do centro do limbo e dos cantos dos olhos pode ser observada nas Figuras 6 (b) e (c) respectivamente.

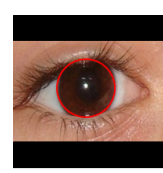

(a)

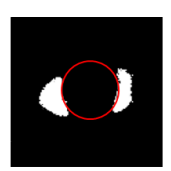

(b)

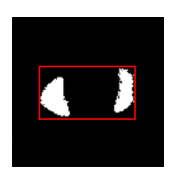

(c)
Fig. 6. (a) Imagem original, (b) Localização do limbo, (c) Localização dos cantos dos olhos. 


\section{E. Detecção do Estrabismo}

As imagens utilizadas no experimento foram adquiridas através do teste de Hirschberg, neste teste, é necessária uma taxa de conversão para obter o ângulo estrábico (em dioptrias de prisma $\Delta$ ) a partir da deslocação observada do reflexo da córnea (em milímetros) [29]. No entanto, o método proposto utiliza o centro dos cantos dos olhos e o centro do limbo como parâmetros de verificação do alinhamento dos olhos para detectar o estrabismo.

Nesse teste, deve-se considerar que um olho é o fixador, ou seja, tem a imagem alinhada com o centro óptico e o outro olho é o que será analisado.

Outra referência que deve ser examinada é o ângulo kappa, pois essa variável precisa ser levada em consideração para identificar o olho que possui desvio. A verificação dos posicionamentos em cada um dos olhos, é obtida primeiramente medindo a distância do centro dos cantos dos olhos para o centro do limbo na horizontal denominando Desvio Horizontal (DHp), isso é expresso pela Equação 14:

$$
D H p=\left|x_{c}-x_{l}\right|
$$

onde $x_{c}$ e $x_{l}$ são respectivamente coordenadas do centro dos cantos dos olhos e centro do limbo de cada olho em pixels e DHp é o valor do desvio horizontal.

Em seguida, utiliza-se o valor médio do diâmetro horizontal do limbo convertendo de pixel para milímetro (pixelMM), levando em consideraçõa o valor médio do diâmetro do limbo de um adulto igual a $11 \mathrm{~mm}$ [30], como mostra a Equação 15.

$$
D H m m=D H p * \operatorname{pixel} M M
$$

Para obter a medida do desvio horizontal do paciente, em seguida, realiza-se a conversão de milímetros para dioptrias primáticas ( $\Delta$ - unidade de medida utilizada pelo especialista). Esse cálculo é expresso pela seguinte Equação 16.

$$
D H \Delta=D P * D H m m
$$

onde $D P$ representa uma constate igual a $15 \Delta$ [31].

\section{REsultados E Discussões}

Esta seção apresenta e discute os resultados alcançados utilizando a metodologia proposta. Serão utilizadas as métricas sensibilidade, especificidade, acurácia e dice [32] para validar os experimentos realizados.

Após a segmentação da esclera utilizando os índices de diversidade filogenética para extração de características e SVM para classificação pixel a pixel dos patches, em seguida para reconstrução das imagens utilizou-se 151 imagens para treinamento e 50 imagens para validação da base Proença and Alexandre (2005) [6]. Sua arquitetura foi construída com a função de ativação RELU e foram definidas 60 épocas. A Figura 7 (b) apresenta o resultado da segmentação utilizando os índices de diversidade filogenética e a Figura 7 (c) apresenta o resultado da rede ResNet e a Tabela I apresenta os resultados da segmentação inicial da esclera e após a reconstrução.

Com isso, para a etapa posterior, foram consideras apenas as imagens que resultaram em pelo menos uma área da esclera
TABELA I

COMPARATIVO ENTRE A SEGMETAÇÃO INICIAL A RECONSTRUÇÃO DA ESCLERA.

\begin{tabular}{ccccc} 
& Dice & Sensibilidade & Especificidade & Acurácia \\
\hline Segmentacação inicial & $63,5 \%$ & $57,2 \%$ & $89,1 \%$ & $89,7 \%$ \\
\hline Reconstrução & $79,5 \%$ & $77 \%$ & $93,5 \%$ & $92,3 \%$ \\
\hline
\end{tabular}

segmentada, pois com sua ausência não e possível localizar os cantos dos olhos.

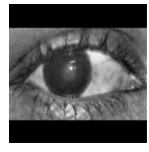

(a)

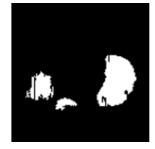

(b)

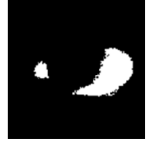

(c)

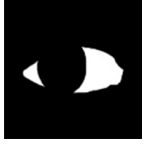

(d)
Fig. 7. (a) - Imagem após o pré-processamento, (b) Segmentação inicial, (c) Reconstrução da esclera, (d) Marcação.

Com o centro dos cantos dos olhos e o centro do limbo, ambos localizados, nos testes realizados, obtivemos uma taxa de acerto de $91 \%$ na localização do canto dos olhos e na localização do limbo $69 \%$ de acurácia, 70,1\% de epecificidade e $67 \%$. O método falhou quando aplicado em imagens que os olhos estão entreabertos e imagens que possuem flash. Por essa razão, para a etapa posterior foram consideras apenas 310 imagens.

De acordo com os estudos de Choi and Kushner (1998) [33] ao analisar a detecção do estrabismo, estimaram que os desvios avaliados pelo especialista divergem entre $5 \Delta$ a $10 \Delta$. Portanto, a partir dos resultados encontrados utilizou-se a tolerância ao erro de $\pm 10 \Delta$ para avaliar o desvio estimado pelo método em relação à medida do especialista. A Tabela II apresenta os resultados obtidos pelo método proposto comparados com a medida do desvio realizada pelo especialista baseado no teste de Hirschberg, bem como a variância e o desvio padrão do erro médio.

Após a localização do centro do limbo e localização do centro dos cantos dos olhos, verificou-se que se o paciente tem um desvio convergente (quando o olho se move para dentro) ou divergente (quando o olho o olho se move para fora) o método quantifica-o com um desvio maior do que possui, sendo esta uma limitação do método, por isso, apenas 28 pacientes (23 com estrabismo e 5 normais) foram levados em consideração nos experimentos.

TABELA II

RESULTADOS DO MÉTODO PROPOSTO PARA AS CINCO POSIÇÕES DO OLHAR.

\begin{tabular}{ccccccc} 
& & & & & & \\
& Imagens & Sensibilidade & Especificidade & Acurácia & Variância & Desvio Padrão \\
\hline DEXTRO & 22 & $87,4 \%$ & $100 \%$ & $89,7 \%$ & 22,5 & 4,47 \\
\hline INFRA & 26 & $84,4 \%$ & $60 \%$ & $81,1 \%$ & 31,5 & 5,62 \\
\hline LEVO & 25 & $70 \%$ & $100 \%$ & $73,5 \%$ & 31,9 & 5,75 \\
\hline PPO & 24 & $90,1 \%$ & $100 \%$ & $91,1 \%$ & 12,7 & 3,57 \\
\hline SUPRA & 23 & $83,9 \%$ & $100 \%$ & $86,1 \%$ & 24,3 & 4,93 \\
\hline
\end{tabular}

A partir dos resultados encontrados na Tabela II pode-se afirmar que o método independe do conjunto de dados em estudo, apresentando o melhor resultado na poição PPO com 
90,1\% de senibilidade, $100 \%$ de epecificidade e $91,1 \%$ de acurácia, se comparado com os demais, pois há um equilíbrio entre as três métricas, ressaltando que os casos de erros são aqueles em que as etapas anteriores não foram tão precisas, alterando assim seu resultado final.

\section{CONCLUSÃo}

Este trabalho apresentou um novo método para detectar automaticamente o estrabismo, avaliando o posicionamento do centro do limbo em relação ao centro dos cantos dos olhos utilizando os índices de diversidade filogenética. Apesar de não ser um método comum utilizado pelos especialistas, pode' se afirmar que o método apresenta um desempenho promissor para deteç̧ão do estrabismo, não apenas em imagens do teste de Hirschberg, mas também em outros exames se a metodologia acertar em todas as etapas.

Assim, o melhor resultado encontrado foi na posição PPO, obtendo sensibilidade de 90,\%, especificidade de $100 \%$ e acurácia de $91,1 \%$. Observa-se também que a etapa de localização precisa dos cantos dos olhos e do limbo são imprescindíveis para a detecção e quantificação correta do desvio. Portanto, pretende-se melhorar essas etapas intermediárias para melhorar os resultados e demonstrar a eficácia da metodologia.

\section{AgRADECIMENTOS}

Os autores agradecem a Coordenação de Aperfeiçoamento de Pessoal de Nível Superior (CAPES).

\section{REFERÊNCIAS}

[1] J. D. S. de Almeida, A. C. Silva, J. A. M. Teixeira, A. C. Paiva, and M. Gattass, "Surgical planning for horizontal strabismus using support vector regression," Computers in biology and medicine, vol. 63, pp. 178-186, 2015.

[2] T. L. A. Valente, J. D. S. de Almeida, A. C. Silva, J. A. M. Teixeira, and M. Gattass, "Automatic diagnosis of strabismus in digital videos through cover test," Computer methods and programs in biomedicine, vol. 140, pp. 295-305, 2017.

[3] Y. Zheng, H. Fu, B. Li, and W.-L. Lo, "An automatic stimulus and synchronous tracking system for strabismus assessment based on cover test," in 2018 International Conference on Intelligent Informatics and Biomedical Sciences (ICIIBMS), vol. 3. IEEE, 2018, pp. 123-127.

[4] M. W. Seo, H. K. Yang, J. M. Hwang, and J.-M. Seo, "The automated diagnosis of strabismus using an infrared camera," in 6th European Conference of the International Federation for Medical and Biological Engineering. Springer, 2015, pp. 142-145.

[5] J. D. Sousa de Almeida, A. C. Silva, J. A. M. Teixeira, A. C. Paiva, and M. Gattass, "Computer-aided methodology for syndromic strabismus diagnosis," Journal of Digital Imaging, vol. 28, no. 4, pp. 462-473, Aug 2015. [Online]. Available: https://doi.org/10.1007/s10278-014-9758-0

[6] H. Proença and L. A. Alexandre, "Ubiris: A noisy iris image database," in International Conference on Image Analysis and Processing. Springer, 2005, pp. 970-977.

[7] A. Das, U. Pal, M. A. F. Ballester, and M. Blumenstein, "Multi-angle based lively sclera biometrics at a distance," in 2014 IEEE Symposium on Computational Intelligence in Biometrics and Identity Management (CIBIM). IEEE, 2014, pp. 22-29.

[8] J. Lu, J. Feng, Z. Fan, L. Huang, C. Zheng, and W. Li, "Automated strabismus detection based on deep neural networks for telemedicine applications," arXiv preprint arXiv:1809.02940, 2018.

[9] D. R. Lucio, R. Laroca, E. Severo, A. S. Britto Jr, and D. Menotti, "Fully convolutional networks and generative adversarial networks applied to sclera segmentation," CoRR, vol. abs/1806.08722, 2018.
[10] D. Riccio, N. Brancati, M. Frucci, and D. Gragnaniello, "An unsupervised approach for eye sclera segmentation," in Iberoamerican Congress on Pattern Recognition. Springer, 2017, pp. 550-557.

[11] M. De Marsico and D. Riccio, "A new data normalization function for multibiometric contexts: A case study," in International Conference Image Analysis and Recognition. Springer, 2008, pp. 1033-1040.

[12] A. O. de Carvalho Filho, A. C. Silva, A. C. de Paiva, R. A. Nunes, and M. Gattass, "Computer-aided diagnosis of lung nodules in computed tomography by using phylogenetic diversity, genetic algorithm, and svm," Journal of digital imaging, vol. 30, no. 6, pp. 812-822, 2017.

[13] — "Lung-nodule classification based on computed tomography using taxonomic diversity indexes and an svm," Journal of Signal Processing Systems, vol. 87, no. 2, pp. 179-196, 2017.

[14] _ "Classification of patterns of benignity and malignancy based on ct using topology-based phylogenetic diversity index and convolutional neural network," Pattern Recognition, vol. 81, pp. 200-212, 2018.

[15] F. S. S. de Oliveira, A. O. de Carvalho Filho, A. C. Silva, A. C. de Paiva, and M. Gattass, "Classification of breast regions as mass and nonmass based on digital mammograms using taxonomic indexes and svm," Computers in biology and medicine, vol. 57, pp. 42-53, 2015.

[16] O. d. S. Santos, T. d. O. Simões, L. N. Mesquita, A. D. d. Sousa, and A. O. d. Carvalho Filho, "Análise filogenética para diferenciação entre nódulos malignos e benignos," J. health inform, vol. 8, no. supl. I, pp. 1061-1070, 2016.

[17] J. Izsák and L. Papp, "A link between ecological diversity indices and measures of biodiversity," Ecological Modelling, vol. 130, no. 1-3, pp. $151-156,2000$

[18] K. Clarke and R. Warwick, "A taxonomic distinctness index and its statistical properties," Journal of applied ecology, vol. 35, no. 4, pp. 523-531, 1998

[19] K. R. Clarke, R. Gorley, P. Somerfield, and R. Warwick, Change in marine communities: an approach to statistical analysis and interpretation. Primer-E Ltd, 2014

[20] G. PIEŃKOWSKI and E. WESTWALEWICZ-MOGILSKA, "Trace fossils from the podhale flysch basin, poland-an example of ecologicallybased lithocorrelation," Lethaia, vol. 19, no. 1, pp. 53-65, 1986.

[21] R. I. Vane-Wright, C. J. Humphries, and P. H. Williams, "What to protect?-systematics and the agony of choice," Biological conservation, vol. 55, no. 3, pp. 235-254, 1991.

[22] M. Keith, C. Chimimba, B. Reyers, and A. Van Jaarsveld, "Taxonomic and phylogenetic distinctiveness in regional conservation assessments: a case study based on extant south african chiroptera and carnivora," in Animal Conservation forum, vol. 8, no. 3. Cambridge University Press, 2005, pp. 279-288.

[23] D. P. Faith, "Conservation evaluation and phylogenetic diversity," Biological conservation, vol. 61, no. 1, pp. 1-10, 1992.

[24] C. O. Webb, "Exploring the phylogenetic structure of ecological communities: an example for rain forest trees," The American Naturalist, vol. 156, no. 2, pp. 145-155, 2000.

[25] K. He, X. Zhang, S. Ren, and J. Sun, "Deep residual learning for image recognition," in Proceedings of the IEEE conference on computer vision and pattern recognition, 2016, pp. 770-778.

[26] K. Ehsani, R. Mottaghi, and A. Farhadi, "Segan: Segmenting and generating the invisible," in Proceedings of the IEEE Conference on Computer Vision and Pattern Recognition, 2018, pp. 6144-6153.

[27] S. Ioffe and C. Szegedy, "Batch normalization: Accelerating deep network training by reducing internal covariate shift," arXiv preprint arXiv:1502.03167, 2015.

[28] R. O'DUDA, "Use of hough transformation to detect lines and curves in picture," CACM, vol. 15, pp. 11-15, 1972.

[29] S. Hasebe, H. Ohtsuki, R. Kono, and Y. Nakahira, "Biometric confirmation of the hirschberg ratio in strabismic children." Investigative ophthalmology \& visual science, vol. 39, no. 13, pp. 2782-2785, 1998.

[30] C. Khng and R. H. Osher, "Evaluation of the relationship between corneal diameter and lens diameter," Journal of Cataract \& Refractive Surgery, vol. 34, no. 3, pp. 475-479, 2008.

[31] G. S. Schwartz, The eye exam: a complete guide. Slack Incorporated, 2006.

[32] L. R. Dice, "Measures of the amount of ecologic association between species," Ecology, vol. 26, no. 3, pp. 297-302, 1945.

[33] R. Y. Choi and B. J. Kushner, "The accuracy of experienced strabismologists using the hirschberg and krimsky tests," Ophthalmology, vol. 105, no. 7, pp. 1301-1306, 1998. 